\title{
TGFB1 +869 T>C (RS1800470) VARIANT IS INDEPENDENTLY ASSOCIATED WITH SUSCEPTIBILITY, LABORATORY ACTIVITY, AND TGF- $\beta 1$ IN PATIENTS WITH SYSTEMIC LUPUS ERYTHEMATOSUS
}

Nicole Perugini Stadtlober ${ }^{1}$, Tamires Flauzino ${ }^{1}$, Lorena Flor da Rosa Franchi Santos ${ }^{1}$, Tatiana Mayumi Veiga Iriyoda², Neide Tomimura Costa ${ }^{1}$, Marcell Alysson Batisti Lozovoy ${ }^{1}$, Edna Maria Vissoci Reiche ${ }^{1}$, Andréa Name Colado Simão ${ }^{1}$,

1.Universidade Estadual de Londrina, Londrina (PR), Brazil; 2.Pontifícia Universidade Católica do Paraná, Londrina (PR), Brazil.

*Corresponding author: tatimayumi54@gmail.com

\section{BACKGROUND}

The systemic lupus erythematosus (SLE) is a complex autoimmune disease represented by alterations in innate and adaptive immune responses leading to inflammation and tissue damage. The etiology of SLE involves several predisposing factors, including genetic, environmental, and hormonal factors. Cytokines also play an important role in the pathophysiology of the disease, through the modulation of immune responses and inflammatory reactions. Growing evidence has demonstrated that genetic variants in the coding and promoting regions of cytokine genes could affect the cytokine production and, consequently, may influence disease susceptibility and clinical manifestations. Thus, the aim of this study was to evaluate the association of the $+869 \mathrm{~T}>\mathrm{C}$ ( $\mathrm{rs} 1800470$ ) and - 509 C > T (rs1800469) TGFB1 genetic variants, individually or in haplotypes structure, with susceptibility, autoantibodies, disease activity, and TGF- $\beta 1$ plasma levels in patients with SLE.

\section{METHODS}

The study included 203 patients with SLE and 165 healthy controls. The TGFB1 variants were determined by real-time polymerase chain reaction (qPCR). Plasma levels of TGF-1 were determined using immunofluorimetric assay.

\section{RESULTS}

Patients and controls did not differ in sex $(p=0.456)$ and ethnicity $(p=0.061)$. However, SLE patients were older ( $p=0.008)$ and had a higher BMI ( $p<0.001)$ than controls. The median disease duration was 10 years (4-15) and only 54 (27.0\%) patients had moderate and high disease activity (SLEDAI $\geq 6$ ). Most SLE patients had positive anti-dsDNA (58.9\%), corticotherapy was the most common treatment (88\%), followed by antimalarials (74.5\%). The TGFB1 + 869 CC genotype was associated with SLE susceptibility $(\mathrm{OR}=1.710,95 \% \mathrm{Cl}=1.020-2.866, p=0.042)$ and with reduction of $\mathrm{C} 4(\mathrm{p}=0.040)$ and TGF- $\beta 1$ levels $(p=0.044)$. In addition, patients with TGFB1 + 869 TC and CC genotypes and positive anti-dsDNA had lower TGF- $\beta 1$ levels than those with TT $(p=0.004)$. The TGFB1 - 509 TT genotype was associated with reduced levels of $C 4(p=0.032)$. There was no association between haplotypes and clinical and laboratory parameters.

\section{CONCLUSION}

Our data demonstrated that the TGFB1 + 869 T > C variant can be used as a genetic marker for SLE susceptibility and both variants as predictors of laboratory activity. This is the first study to demonstrated that TGF- 1 levels can be modulated by the interaction between TGFB1 + 869 C allele, in homozygosity, or heterozygosity, and the presence of anti-dsDNA.

\section{KEYWORDS}

Systemic lupus erythematosus, Transforming growth factor-beta 1, rs1800470, rs1800469, SLEDAI. 